preprint DO-TH-95/10

hep-ph/9507285

July 11th 1995

\title{
A dynamical model for nuclear structure functions
}

\author{
D. Indumathi and W. Zhu円 \\ Institut für Physik, Universität Dortmund, Germany
}

\begin{abstract}
The dynamical origins of the EMC effect are studied. We conclude that a swelling in size of a bound nucleon as well as nuclear binding plays an important rôle in determining the parton distributions within a bound nucleon. We find that the distortion of nucleon structure functions in nuclei can be simply explained with a few fundamental nuclear parameters.
\end{abstract}

PACS numbers: 13.60.Hb, 24.85.+p, 21.10.Dr

The fact that the structure functions of bound and free nucleons are not equal is called the EMC effect [1]. Recent accurate data [2, 3, 4] on nuclear structure functions impels us to reconsider the origin of the EMC effect. In this letter we report that this effect can be explained in a broad kinematical region using the idea of swelling and incorporating binding effects using only a few fundamental nuclear parameters but with a new understanding concerning these concepts. We expound on our ideas as follows.

As the first step, we choose a set of parton distributions which can describe the structure functions of the free nucleon in the kinematic region of the EMC effect (where $Q^{2}$ ranges from less than $1 \mathrm{GeV}^{2}$ to a hundred $\mathrm{GeV}^{2}$ ). One such model was proposed by Glück, Reya, and Vogt [5]. The model assumes that there exists a scale $Q^{2}=\mu^{2}$ which separates the perturbative regime $\left(Q^{2} \geq \mu^{2}\right)$ from the nonperturbative one $\left(Q^{2}<\Lambda^{2}\right)$. All parton distributions are generated dynamically by evolution from a set of valence-like inputs (of the form $N x^{\alpha} P_{N, q}(x)(1-x)^{\beta}$ ) at $\mu^{2}$. For example, in the leading order (LO) approximation with $\mu^{2}=0.23 \mathrm{GeV}^{2}$, the valence $\left(u_{v}, d_{v}\right)$, the total sea $(S)$, and gluon $(g)$ input distributions are given to be [5],

$$
\begin{aligned}
x u_{v}^{N}(x) & =1.377 x^{0.549} P_{N, u}(x)(1-x)^{3.027}, \\
x d_{v}^{N}(x) & =0.328 x^{0.366} P_{N, d}(x)(1-x)^{3.744}, \\
x S_{N}(x) & =2 x(\bar{u}+\bar{d})=2.40 x^{0.29} P_{N, S}(x)(1-x)^{7.88}, \\
x g_{N}(x,) & =35.8 x^{2.3}(1-x)^{4.0},
\end{aligned}
$$

\footnotetext{
${ }^{1}$ On leave from the Department of Physics, East China Normal University, Shanghai 200062, P. R. China
} 
where

$$
\begin{aligned}
& P_{N, u}(x)=1+0.81 \sqrt{x}-4.36 x+19.4 x^{3 / 2}, \\
& P_{N, d}(x)=1+1.14 \sqrt{x}+5.71 x+16.9 x^{3 / 2}, \\
& P_{N, S}(x)=1+0.31 x .
\end{aligned}
$$

The shape of the valence quark distributions in (1) is similar to that of the gluons, while the input sea quarks are much softer. This can be understood if we assume that the gluons are co-moving with the valence quarks whereas the sea quarks are mesonic at $Q^{2}=\mu^{2}$, i.e., the input sea quarks correspond to the mesonic component of the nucleon at $Q^{2}<\Lambda^{2}$, so that their distribution will be softened by a convolution form [6].

We now discuss the nuclear binding effect at $Q^{2}=\mu^{2}$. Fermi motion is a global property of the bound nucleon. We regard the energy of Fermi motion as a part of the effective mass of the bound nucleon. This does not change the total momentum of the nucleon but only smears the parton distributions in the large $x$ region. Since we are interested here primarily in the small and intermediate $x$ region, we do not consider this effect in what follows. Therefore, the momentum of the struck nucleon in the lab frame is $P_{N}=\left(M_{N}-2 b, \mathbf{p}\right)$, where $b$ is the binding energy per nucleon. The factor 2 is due to the fact that the nucleus with mass $(A-1)$ is required to be on mass-shell. Our approach is different from traditional models of the EMC effect [7] since we do not need to compensate for the binding energy. Instead, we try by using the Weizsäcker mass formula [8] to establish the connection between the binding effect and parton distributions in nuclei. According to this formula, the binding energy per nucleon arising strictly from the nuclear force is

$$
b=\left[1-P_{s}(A)\right] a_{\mathrm{vol}}+P_{s}(A) \frac{a_{\mathrm{vol}}}{2}=a_{\mathrm{vol}}-a_{\mathrm{sur}} A^{-1 / 3},
$$

for $A>12$ where $P_{s}(A)$ is the probability of finding a nucleon on the nuclear surface. We have ignored other contributions, especially the Coulombic one, which is not probed in DIS. Experimentally $a_{\mathrm{vol}}=15.67 \mathrm{MeV}$, and $a_{\mathrm{sur}}=17.23 \mathrm{MeV}$.

On the other hand, the attractive potential describing the nuclear force is from the exchange of scalar mesons. The energy required for this nuclear binding is taken away from individual nucleons which thus lose energy. We assume that the binding energy, $b$, corresponds to loss of energy of "mesonic" sea quarks in the nucleons. This means that the momentum fraction carried by the sea quarks in a nucleon bound in a nucleus at $Q^{2}=\mu^{2}$ will be reduced to

$$
\begin{aligned}
S_{A}\left(x, \mu^{2}\right) & =K(A) S_{N}\left(x, \mu^{2}\right) \\
& =\left(1-\frac{2 b}{M_{N}\left\langle S_{N}\left(\mu^{2}\right)\right\rangle_{2}}\right) S_{N}\left(x, \mu^{2}\right) .
\end{aligned}
$$

Here $\left\langle S_{N}\right\rangle_{2}$ is the momentum fraction (second moment) of the sea quarks and we assume that the decrease in number of sea quarks due to the binding effect is proportional to the quark density.

A bound nucleon will physically swell in size and this has been discussed in the socalled rescaling models, in which the rescaling of the input point, $\mu^{2}$, is assumed [9]. 
However, we consider that the swelling of the nucleon only geometrically redistributes partons inside the nucleon and does not change either the value of this dynamical parameter or the existing number of partons at $\mu^{2}$. We try to describe the swelling effect using some universal principles as in [10]. The relative increase in the nucleon's radius is $\delta_{A}$, where $\left(R_{N}+\Delta R(A)\right) / R_{N}=1+\delta_{A}$. Analogous to (2), we assume that the swelling of a nucleon on the nuclear surface is less than that of one in the interior; therefore,

$$
\delta_{A}=\left[1-P_{s}(A)\right] \delta_{\mathrm{vol}}+P_{s}(A) \delta_{\mathrm{vol}} / 2 .
$$

Here $\delta_{\text {vol }}$ parametrises the swelling of the nucleon in the interior of a heavy nucleus and is a constant for nuclei with $A>12$ and also for Helium (with $P_{s}=1$ ) since they have similar nuclear densities. Interestingly, the distortions of the density distributions and hence the changes in the three main parameters, $N, \alpha$, and $\beta$, in (1), due to swelling (if we assume $P_{A, q}(x)=P_{N, q}(x)$ ), can be simply determined by some universal principles. These cause the first three moments of the parton distributions in a free $\left(q_{N}\right)$ and bound $\left(q_{A}\right)$ nucleon (for $q=$ valence, sea quarks, and gluons) at $\mu^{2}$ to be related by

$$
\begin{aligned}
\left\langle q_{A}\left(\mu^{2}\right)\right\rangle_{1} & =\left\langle q_{N}\left(\mu^{2}\right)\right\rangle_{1}, \\
\left\langle q_{A}\left(\mu^{2}\right)\right\rangle_{2} & =\left\langle q_{N}\left(\mu^{2}\right)\right\rangle_{2}, \\
\frac{\left(\left\langle q_{N}\left(\mu^{2}\right)\right\rangle_{3}-\left\langle q_{N}\left(\mu^{2}\right)\right\rangle_{2}^{2}\right)^{1 / 2}}{\left(\left\langle q_{A}\left(\mu^{2}\right)\right\rangle_{3}-\left\langle q_{A}\left(\mu^{2}\right)\right\rangle_{2}^{2}\right)^{1 / 2}} & =1+\delta_{A} .
\end{aligned}
$$

The first two equations imply number and momentum conservation of partons and the last incorporates the swelling effect [10]. Fig. 1 gives an example of the swelling effect for the parton densities in calcium. The momenta lost from the small and large $x$ regions are transferred to the intermediate $x$ region. The effect thus weakly enhances the distributions in the region $0.1<x<0.3$ and results in "antishadowing". Furthermore, this enhancement depends on the nuclear density and does not disappear at larger $Q^{2}$.

Finally, there is a further depletion of the sea densities at the time of scattering. This is easiest to see in the Breit frame, where the exchanged virtual boson is completely space-like, so that the 3-momentum of the struck parton is flipped in the interaction. Hence, a struck parton carrying a fraction $x$ of the nucleon's momentum, $P_{N}$, during the interaction time $\tau_{\text {int }}=1 / \nu$, will be localized longitudinally to within a potentially large distance $\Delta Z \sim 1 /\left(2 x P_{N}\right)$. Although the spatial extent of a single colored parton cannot exceed the range of QCD confinement, one can assume that a struck parton with $x<x_{0}=1 /\left(2 M_{N} d_{N}\right)$ can combine with a wee parton and form a colorless state $T_{0}$ with vacuum quantum numbers. Here $d_{N}$ is the average correlation distance between two neighboring nucleons in the lab frame:

$$
\begin{aligned}
d_{N}=P_{s}(A)\left[R_{N}+2\left(R_{\mathrm{WS}}-R_{N}\right)\right]+ \\
\left(1-P_{s}(A)\right)\left[\frac{R_{N}}{2}+2\left(R_{\mathrm{WS}}-R_{N}\right)\right],
\end{aligned}
$$

where $R_{N}$ and $R_{\mathrm{WS}}$ are the nucleon and Wigner-Staiz radii. The observable value of the momentum of $T_{0}$ is its average over the uncertainty time $\tau_{\text {int }}$ and equals zero in the Breit frame. Therefore, $T_{0}$ is a static scalar field. 


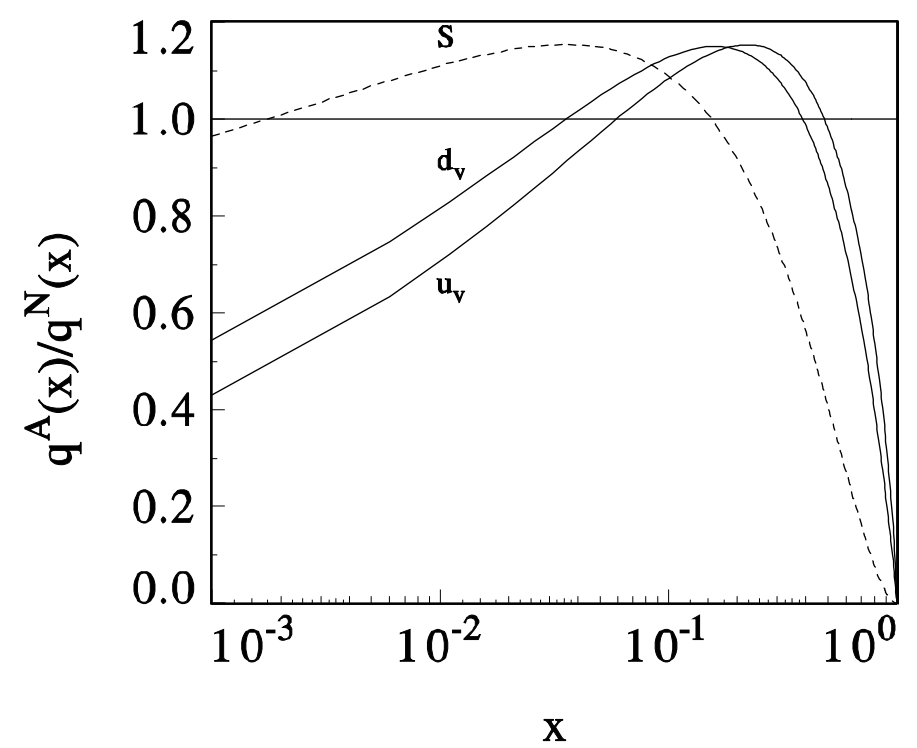

Fig. 1 The effect of nucleon swelling on the calcium input distributions: the ratios of the modified to unmodified densities are shown for $u_{v}, d_{v}$, and $S$.

We know that the correlation between two nucleons via scalar field exchange is the bound state problem. Therefore, in the DIS process, a nucleon can interact with other nucleons via $T_{0}$ in the long-distance. Because of this, a new binding effect will be experienced by the correlated nucleons, which we call the second binding effect.

Consider a pair of nucleons which are correlated by $T_{0}$. The interaction causes the number of sea quarks $S_{A}\left(x, Q^{2}\right)$ in a nucleon to be further reduced to $S_{A}^{\prime}\left(x, Q^{2}\right)$. (Valence quarks are not depleted due to the requirement of quantum number conservation). Similar to (3), one can simply assume that the loss in number of sea quarks is proportional to the original density, i.e., $S_{A}\left(x, Q^{2}\right)-S_{A}^{\prime}\left(x, Q^{2}\right)=\beta S_{A}\left(x, Q^{2}\right)$, where $\beta$ is a constant. The total energy loss in a bound nucleon due to the second binding effect therefore is

$$
U_{s}\left(Q^{2}\right)=\beta M_{N} \int_{0}^{x_{0}} x S_{A}\left(x, Q^{2}\right) \simeq \beta M_{N}\left\langle S_{A}\left(Q^{2}\right)\right\rangle_{2} .
$$

Hence,

$$
\beta=\frac{U_{s}\left(Q^{2}\right)}{M_{N}\left\langle S_{A}\left(Q^{2}\right)\right\rangle_{2}}=\frac{U\left(\mu^{2}\right)}{M_{N}\left\langle S_{N}\left(\mu^{2}\right)\right\rangle_{2}},
$$

$U\left(\mu^{2}\right)=a_{\mathrm{vol}} / 6$ being the binding energy between each pair of nucleons.

Partons with momentum fraction $x$ can overlap $(n-1)$ other nucleons, where $n=1 /\left(2 M_{N} d_{N} x\right)=x_{0} / x$. The second binding effect arising from each overlap is simply additive due to the applicability of the superposition principle to the linear static field. The corresponding shadowing of the sea quarks is given by $S_{A}^{\prime}\left(x, Q^{2}\right)=$ $K^{\prime}(A) S_{A}\left(x, Q^{2}\right)$, where the depletion factor is,

$$
\begin{aligned}
K^{\prime}(A) & =1, & & \text { when } x>x_{0} ; \\
& =1-2 \beta\left(x_{0} x^{-1}-1\right), & & \text { when } x_{A}<x<x_{0} ; \\
& =1-2 \beta\left(x_{0} x_{A}^{-1}-1\right), & & \text { when } x<x_{A},
\end{aligned}
$$


where $2 \beta=0.037, x_{A}=1 /\left(4 \bar{R}_{A} M_{N}\right)$, and $2 \bar{R}_{A} \simeq 1.4 R_{A}$ is the average thickness of the nucleus. We emphasize that the second binding effect acts on the intermediate state of the probe-target interaction and does not participate in the QCD evolution of the initial state. Since $x_{0} \lesssim 0.1$, this is also a small $x$ effect.

In summary, the input nucleon structure function (for an isoscalar nucleus) is given by

$$
\begin{aligned}
F_{A}\left(x, \mu^{2}\right)=\left\langle e^{2}\right\rangle[ & x u_{v}^{A}\left(x, \mu^{2}\right)+x d_{v}^{A}\left(x, \mu^{2}\right) \\
& \left.+K(A) x S_{A}\left(x, \mu^{2}\right)\right],
\end{aligned}
$$

where the average charge square factor is $\left\langle e^{2}\right\rangle=5 / 18$ and $q^{A}\left(x, \mu^{2}\right)$ incorporates the effect of swelling on every input parton density, $q^{N}\left(x, \mu^{2}\right)$. The structure function ratio of nucleons bound in two different nuclei, $A$ and $B$, at $Q^{2}>\mu^{2}$ is given by,

$$
R^{\mathrm{AB}}=\frac{x u_{v}^{A}\left(x, Q^{2}\right)+x d_{v}^{A}\left(x, Q^{2}\right)+K^{\prime}(A) x \widetilde{S}_{A}\left(x, Q^{2}\right)}{x u_{v}^{B}\left(x, Q^{2}\right)+x d_{v}^{B}\left(x, Q^{2}\right)+K^{\prime}(B) x \widetilde{S}_{B}\left(x, Q^{2}\right)},
$$

where $\widetilde{S}_{A}\left(x, Q^{2}\right)$ corresponds to the evolution of $K(A) S_{A}\left(x, \mu^{2}\right)$, i.e., of the swelled input sea density, depleted by the binding effect, to the scale $Q^{2}$. Note that for $Q^{2}>\mu^{2}$, the sea density also includes a finite contribution from strange quarks. The smearing effect of Fermi motion (at large $x$ ) is neglected in this work.

In fig. 2 we give our predictions for the $x, Q^{2}$, and $A$ dependences of the ratios for $\mathrm{He} / \mathrm{D}, \mathrm{C} / \mathrm{D}$ and $\mathrm{Ca} / \mathrm{D}$. The only free parameter to be fixed in computing these ratios is the value of $\delta_{\mathrm{vol}}$. We find that $\delta_{\mathrm{vol}}=0.15$ best describes the various available data for these nuclei.

We have also shown the ratio of the ${ }^{6} \mathrm{Li}$ to $\mathrm{D}$ structure functions in fig. 2. Unlike helium (which also has $A<12$ ), lithium is a very loosely bound nucleus (whose density is less than half that of helium or carbon, while its radius is larger than that of carbon [2]). Hence, $\delta_{A}$ for Li can be expected to be much smaller than that for He $\left(\delta_{\mathrm{He}}=0.15 / 2\right.$ according to $\left.(4)\right)$ and we take it to be 0.033 . In all cases, the swelling effect dominates the intermediate $x$ ratios while both the binding effects determine the small $x$ ratios.

The ratios $R^{\mathrm{AB}}=F_{2}^{A} / F_{2}^{B}$ for $A / B$ corresponding to $\mathrm{C} / \mathrm{Li}, \mathrm{Ca} / \mathrm{Li}$, and $\mathrm{Ca} / \mathrm{C}$ are more sensitively dependent on the model of the EMC effect (as seen in fig. 3). We predict, in general, an enhancement of the ratio, $R^{\mathrm{AB}}$, at intermediate $x$ which is a net effect of the swelling and the first binding effect, both of which depend on the nuclear density. On the other hand, the strong depletion at small $x$ depends on both the nuclear radius and density. The weaker enhancement of the intermediate $x$ ratios of $\mathrm{Ca}$ and $\mathrm{C}$ is because of their similar densities [11] as compared to the $\mathrm{C} / \mathrm{Li}$ and $\mathrm{Ca} / \mathrm{Li}$ ratios where the two nuclei have different densities. Our predictions are seen to be in excellent agreement with the NMC data [3], also shown for the sake of comparison. Note that we have computed these three ratios at the same values of $\left(x, Q^{2}\right)$ as the data. We emphasize that, apart from the swelling parameter, $\delta_{\mathrm{vol}}$, all parameters are fixed by a few fundamental nuclear parameters. 


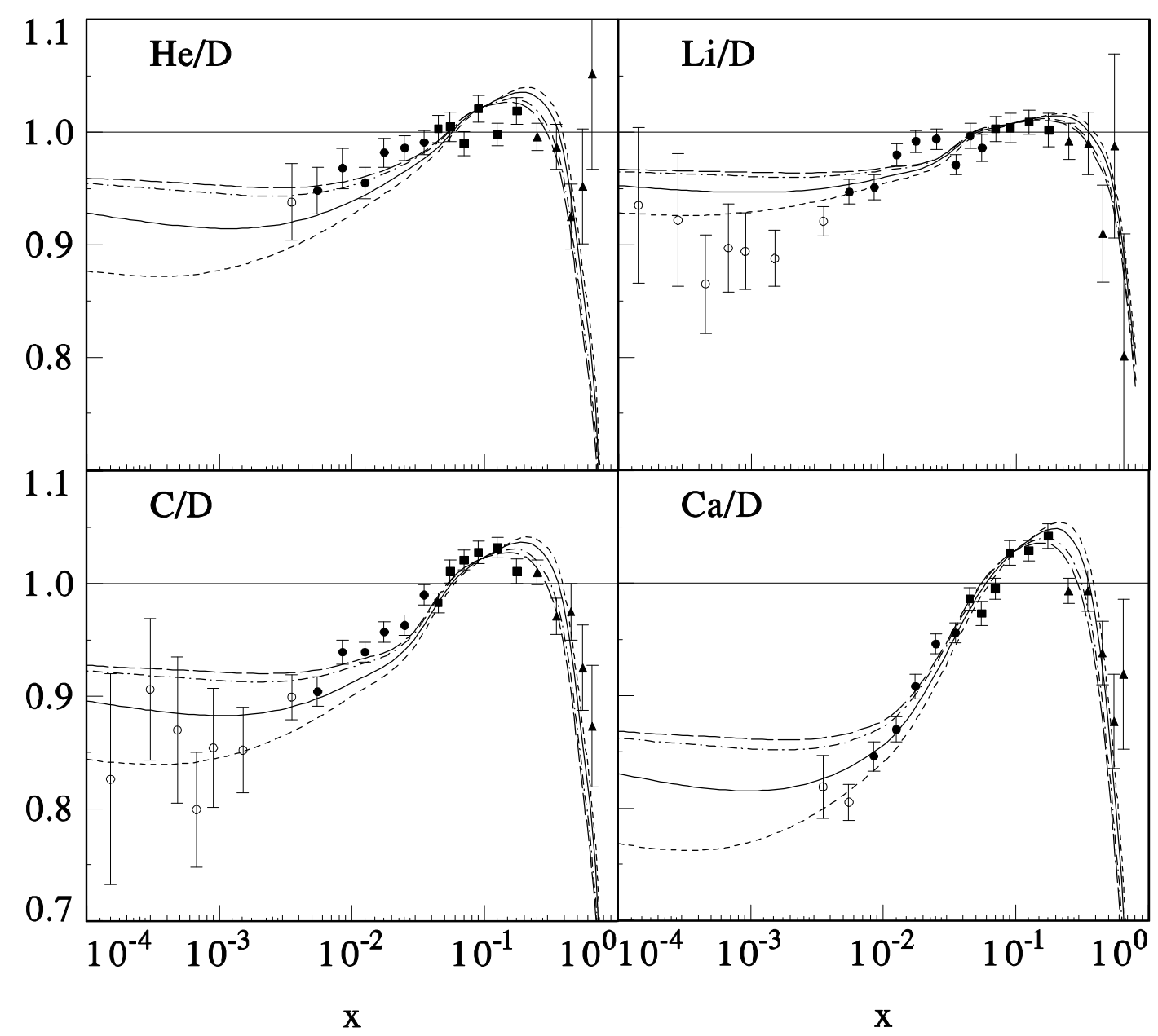

Fig. 2 The structure function ratios as functions of $x$ for (a) $\mathrm{He} / \mathrm{D}$, (b) $\mathrm{Li} / \mathrm{D}$, (c) $C / D$, and (d) $\mathrm{Ca} / \mathrm{D}$. The dashed, full, broken, and long-dashed curves correspond to $Q^{2}=$ $0.5,1,5$, and $15 \mathrm{GeV}^{2}$ respectively. The data, [3, 国, shown as open and solid circles, boxes and triangles correspond to $Q^{2}<1,1-5,5-15$, and $>15 \mathrm{GeV}^{2}$ respectively.

The input (1) reliably predicts the free nucleon stucture function in the region $Q^{2}>0.5 \mathrm{GeV}^{2}$. Hence our dynamical model is also expected to predict correctly, not only the ratios, but also the values of the nuclear structure functions themselves in the same kinematic region. The detailed comparison between the theory and the data will published elsewhere.

In conclusion we have shown that the EMC effect can be explained using swelling and binding effects but with a new understanding of these ideas. As the general properties of the nuclear force originated from the binding effect of nuclei in the history of nuclear physics, we expect that a new understanding of binding effects in the EMC effect will bring to light the nature of the nuclear force at the level of quarks and gluons. 


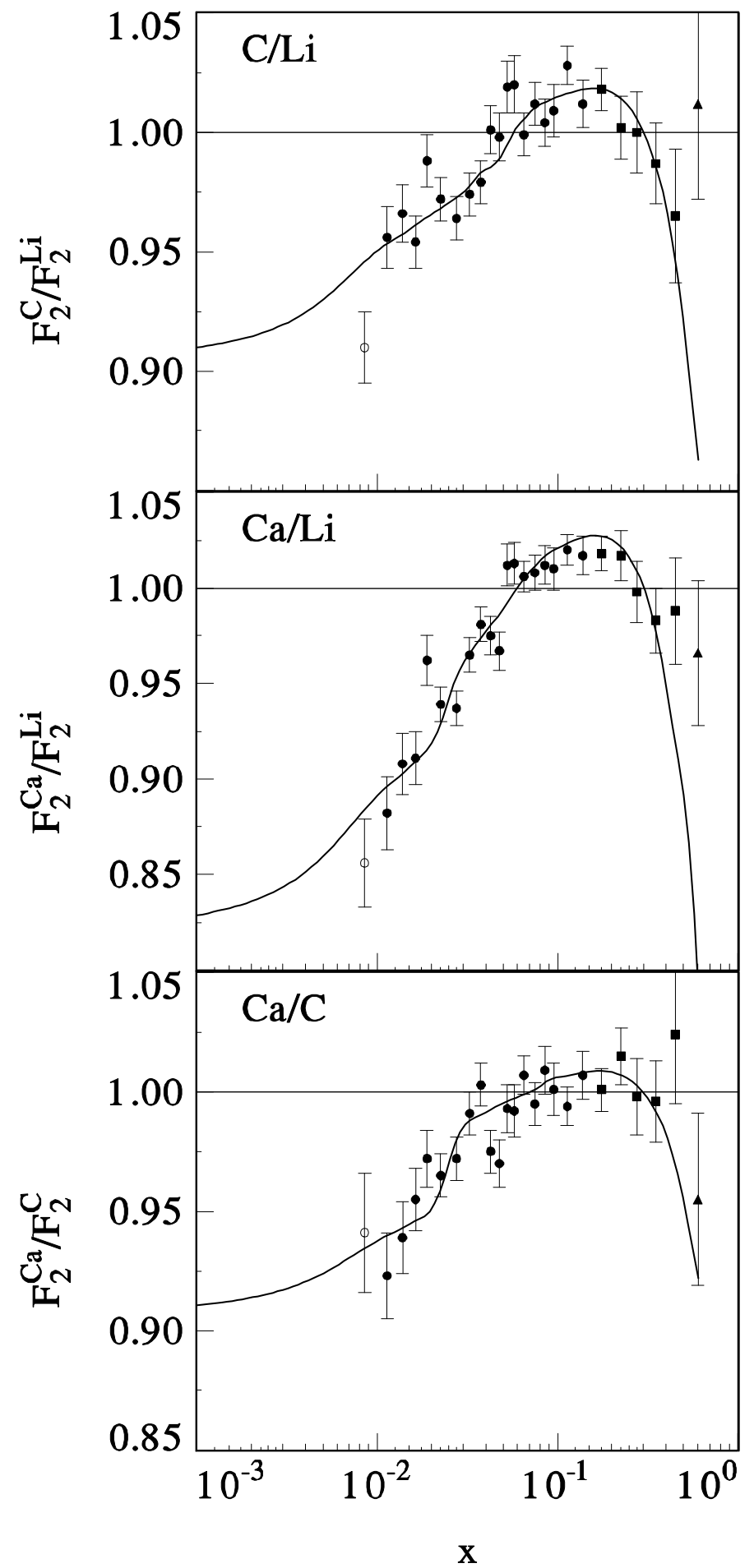

Fig. 3 The structure function ratios for (a) $\mathrm{C} / \mathrm{Li}$, (b) $\mathrm{Ca} / \mathrm{Li}$, and (c) $\mathrm{Ca} / \mathrm{C}$. The solid curve is a smooth fit to our theoretical predictions at the same $\left(x, Q^{2}\right)$ as each available data point [3]. The description of the data is the same as in fig. 2 .

We thank M. Glück and E. Reya for extensive discussions and encouragement. One of us (W. Z) acknowledges the support of the DAAD-K. C. Wong Fellowships 
and the National Natural Science Foundation of China and the hospitality of the University of Dortmund where this work was done.

\section{References}

[1] J. J. Aubert et al., The EMC, Phys. Lett. B 123, 275 (1982); for a recent review, see M. Arneodo, Phys. Rep. 240, 301 (1994).

[2] P. Amaudruz et al., The EMC, Z. Phys. C 53, 73 (1992).

[3] P. Amaudruz et al., The NMC, Nucl. Phys. B 441, 3 (1995).

[4] M. Arneodo et al., The NMC, Nucl. Phys. B 441, 12 (1995).

[5] M. Glück, E. Reya, and A. Vogt, Z. Phys. C 48, 471 (1990); University of Dortmund, Dortmund, Preprint No. DO-TH-94/24, 1994 (to appear in Z. Phys. C).

[6] J. D. Sullivan, Phys. Rev. D 5, 1732 (1972).

[7] M. Ericson and A. W. Thomas, Phys. Lett. B 128, 112 (1983); C. H. Llewellyn Smith, Phys. Lett. B 128, 107 (1983).

[8] C. F. von Weizsäcker, Z. Phys. 96, 431 (1935); H. A. Bethe and R. F. Bacher, Rev. Mod. Phys. 8, 82 (1936).

[9] F. E. Close, R. G. Roberts, and G. G. Ross, Phys. Lett. B 129, 346 (1983); R. L. Jaffe, Phys. Rev. Lett. 50, 228 (1983).

[10] W. Zhu and J. G. Shen, Phys. Lett. B 219, 107 (1989); W. Zhu and L. Qian, Phys. Rev. C 45, 1397 (1992).

[11] The radii and densities, $\left(R_{A} \mathrm{fm}, \rho \mathrm{fm}^{-3}\right)$, of the various nuclei, as quoted in [2], are $(2.6,0.04)$ for ${ }^{6} \mathrm{Li},(2.5,0.09)$ for ${ }^{12} \mathrm{C}$, and $(3.5,0.11)$ for ${ }^{40} \mathrm{Ca}$. 